\title{
Noetherian Varieties in Definably Complete Structures
}

\author{
Tamara Servi
}

\begin{abstract}
We prove that the zero-set of a $C^{\infty}$ function belonging to a noetherian differential ring $M$ can be written as a finite union of $C^{\infty}$ manifolds which are definable by functions from the same ring. These manifolds can be taken to be connected under the additional assumption that every zero-dimensional regular zero-set of functions in $M$ consists of finitely many points. These results hold not only for $C^{\infty}$ functions over the reals, but more generally for definable $C^{\infty}$ functions in a definably complete expansion of an ordered field. The class of definably complete expansions of ordered fields, whose basic properties are discussed in this paper, expands the class of real closed fields and includes o-minimal expansions of ordered fields. Finally, we provide examples of noetherian differential rings of $C^{\infty}$ functions over the reals, containing non-analytic functions.
\end{abstract}

\section{Introduction}

Definable completeness is a weak (first order expressible) version of Dedekind completeness.

1.0.1 Definition. Fix a language $L=\{+,-, \cdot,<, 0, \ldots\}$ which is an expansion of the language of ordered rings. A definably complete structure $\mathbb{K}$ (in the language $L$ ) is an $L$-expansion of an ordered field, such that every definable subset of the domain of $\mathbb{K}$ which is bounded from above, has a least upper bound.

Let $L$ be a language as in Definition 1.0.1. An $L$-expansion of an ordered field is o-minimal if every definable subset of the domain is a finite union of open intervals and points. Hence, every o-minimal $L$-expansion of an ordered field is definably complete. On the other hand, every definably complete structure expands a real closed field (see Theorem 2.0.1). 
Every $L$-expansion of the real ordered field $\mathbb{R}$ is clearly definably complete, by Dedekind completeness. In particular, not every definably complete structure is o-minimal.

The notion of definable completeness is thus weaker than that of ominimality (which has been extensively studied, see for example [1]). However, it has the advantage of being first order expressible. Unlike the ominimal case, the class of all definably complete structures in a given language $L$ is recursively axiomatizable. In particular, if $\mathcal{R}$ is elementary equivalent to an expansion of the real ordered field, then $\mathcal{R}$ is definably complete.

The aim of this paper is to lay the common groundwork for an axiomatic analysis of expansions of the real ordered field with some $C^{\infty}$ functions, such as power functions, the exponential function, the sine function, quasianalytic functions. The goal is to understand the geometric behaviour of sets definable in models of recursively axiomatized fragments of the theories of these structures, and hence to contribute to the solution of decidability questions related to these structures.

The main result of this paper is Theorem 5.0.7, which states that, in a definably complete structure $\mathbb{K}$,

Notice that we do not assume geometric finiteness (o-minimality, Pfaffianity,...), hence this decomposition theorem holds for a wide class of functions over definably complete structures. Over the real numbers, we find analytic but non-tame examples like $\sin (x)$, and in Section 7 we exhibit some $C^{\infty}$ but non-analytic examples.

Results of a similar flavour have been obtained by A. Gabrielov in the context of real analytic functions restricted to a compact ball (see [4]).

This result is a first step in the analysis of quantifier-free definable sets in an expansion of the real field with noetherian functions.

We apply our decomposition theorem to prove a Khovanskii-type finiteness result (see Theorem 6.0.6): given a noetherian differential ring $M$ of functions, if every zero-dimensional regular zero-set of functions in $M$ consists of finitely many points, then the zero-set of any function in $M$ has finitely many connected components.

Finally, we refine the candidate for a complete recursive axiomatization of the real exponential field $\mathbb{R}_{\exp }$ proposed by Macintyre and Wilkie in [8]. We note that the axiom schemes $A 3, A 4, T_{N A}, T_{H}$ in [8] follow from results $2.0 .7,3.0 .7,2.0 .6$ and 2.0 .4 respectively. 


\section{Basic results}

We fix, for the rest of this paper, a language $L$, which expands the language of ordered rings, and a definably complete $L$-structure $\mathbb{K}$. We equip $\mathbb{K}$ with the interval topology, and any power $\mathbb{K}^{n}$ with the product topology.

We give the usual $\varepsilon, \delta$-definition of continuous function (where $\varepsilon$ and $\delta$ are elements of $\mathbb{K}$ ). Limits are well defined, since the topology is Hausdorff.

The following classical results hold true in definably complete structures (the missing proofs are easy and can be found in [14]).

2.0.1 Theorem (Intermediate value). Let $a, b \in \mathbb{K}$ and $f:[a, b] \rightarrow \mathbb{K}$ be $a$ continuous definable function such that $f(a)<0$ and $f(b)>0$. Then there exists $c \in(a, b)$ such that $f(c)=0$.

In particular, every definably complete structure is a real closed field.

2.0.2 Corollary (Intermediate Value Property). Let $a, b \in \mathbb{K}$ and $f$ : $[a, b] \rightarrow \mathbb{K}$ be a continuous definable function. Then $f$ takes all values in $\mathbb{K}$ between $\inf f$ and $\sup f$ (which exist, possibly $\pm \infty$, by definable completeness).

2.0.3 Theorem (Weierstrass Property). Let $a, b \in \mathbb{K}$ and $f:[a, b] \rightarrow \mathbb{K}$ be $a$ continuous definable function. Then $f$ is achieves maximum and minimum on $[a, b]$.

A definable function $f: \mathbb{K} \rightarrow \mathbb{K}$ is differentiable at $x \in \mathbb{K}$ if the limit $\lim _{y \rightarrow x} \frac{f(y)-f(x)}{|y-x|}$ exists. Note that the derivative of a definable function (if it exists) is again a definable function. Analogously, if $f: \mathbb{K}^{n} \rightarrow \mathbb{K}$, we define the partial derivatives. As usual, we say that a definable function $f$ is $C^{1}$ if it is differentiable, with continuous first derivatives. $C^{n}$ and $C^{\infty}$ are similarly defined.

2.0.4 Theorem (Taylor's Theorem). - Let $F: \mathbb{K}^{n} \rightarrow \mathbb{K}$ be a definable $C^{N}$ map and let $\bar{x}_{0}, \bar{x} \in \mathbb{K}^{n}$. Then there exists $\bar{\xi}$, lying on the line segment joining $\bar{x}_{0}$ and $\bar{x}$, such that

$$
\begin{gathered}
F(\bar{x})= \\
F\left(\bar{x}_{0}\right)+F^{\prime}\left(\bar{x}_{0}\right)\left[\bar{x}-\bar{x}_{0}\right]+\frac{F^{\prime \prime}\left(\bar{x}_{0}\right)}{2}\left[\bar{x}-\bar{x}_{0}, \bar{x}-\bar{x}_{0}\right]+\ldots+\frac{F^{(N)}(\bar{\xi})}{N !}\left[\bar{x}-\bar{x}_{0}, \ldots, \bar{x}-\bar{x}_{0}\right]
\end{gathered}
$$

- Let $F: \mathbb{K}^{n} \rightarrow \mathbb{K}^{n}$ be a definable $C^{N+1}$ map and let $\bar{x}_{0}, \bar{x} \in \mathbb{K}^{n}$ such that $\left|\bar{x}-\bar{x}_{0}\right|<r$. Then

$$
\begin{gathered}
\left|F(\bar{x})-F\left(\bar{x}_{0}\right)-F^{\prime}\left(\bar{x}_{0}\right)\left[\bar{x}-\bar{x}_{0}\right]-\ldots-\frac{F^{(N)}\left(\bar{x}_{0}\right)}{N !}\left[\bar{x}-\bar{x}_{0}, \ldots, \bar{x}-\bar{x}_{0}\right]\right| \leq \\
\sup _{\bar{y} \in B\left(\bar{x}_{0}, r\right)}\left|\frac{F^{(N+1)}(\bar{y})}{(N+1) !}\left[\bar{x}-\bar{x}_{0}, \ldots, \bar{x}-\bar{x}_{0}\right]\right|
\end{gathered}
$$


2.0.5 Corollary (Increasing functions and the sign of the derivative). Let $a, b \in \mathbb{K}$ and $f:(a, b) \rightarrow \mathbb{K}$ be a $C^{1}$ definable function. If for all $x \in(a, b)$ we have $f^{\prime}(x)>0$, then $f$ is strictly increasing on $(a, b)$.

Let $F: \mathbb{K}^{n} \rightarrow \mathbb{K}^{n}$ be a $C^{2}$ definable map. Suppose we are given some point $\bar{x}_{0} \in \mathbb{K}^{n}$ such that $\left|F\left(\bar{x}_{0}\right)\right|$ is small, $\left|F^{\prime}\left(\bar{x}_{0}\right)\right|$ is bounded away from zero, and $\left|F^{\prime}(\bar{x})\right|$ and $\left|F^{\prime \prime}(\bar{x})\right|$ are not too large on a suitable neighbourhood of $\bar{x}_{0}$. Then $F$ has a zero, moreover a nonsingular one, lying near to $\bar{x}_{0}$. More precisely,

2.0.6 Theorem (Newton's Method). Let $a_{0}, a_{1}, a_{2} \geq 1$. Then there exist $m, r \in \mathbb{K}^{+}$(which can be written as rational functions of $n, a_{0}, a_{1}, a_{2}$ ) such that, $\forall \bar{x}_{0} \in \mathbb{K}^{n}$,

If $\quad\left|F\left(\bar{x}_{0}\right)\right|<m$ and

$\forall \bar{y} \in B\left(\bar{x}_{0}, r\right)\left|F^{\prime}(\bar{y})^{-1}\right|<a_{0}$ and $\left|F^{\prime}(\bar{y})\right|<a_{1}$ and $\left|F^{\prime \prime}(\bar{y})\right|<a_{2}$,

Then $\exists \bar{x} F(\bar{x})=0$ and $\bar{x} \in B\left(\bar{x}_{0}, r\right)$.

The proof is based on a repeated use of Taylor's Theorem.

Proof. Let $r=\left(2 n^{3} a_{0}^{2} a_{1} a_{2}\right)^{-1}$ and $m=\left(4 n^{3} a_{0}^{3} a_{1} a_{2}\right)^{-1}$.

Let $\bar{x} \in B\left(\bar{x}_{0}, r\right)$ be such that $|F(\bar{x})|=\min \left\{|F(\bar{u})|: \bar{u} \in B\left(\bar{x}_{0}, r\right)\right\}$ (the existence of such a point $\bar{x}$ follows from the fact that the function $\bar{u} \mapsto|F(\bar{u})|$ is continuous definable). We claim $F(\bar{x})=0$. Let

$$
\bar{y}=\bar{x}-F^{\prime}(\bar{x})^{-1} \cdot F(\bar{x}) .
$$

Equivalently, $F(\bar{x})=F^{\prime}(\bar{x})[\bar{x}-\bar{y}]$. It is sufficient to show that:

(i) $\bar{y} \in B\left(\bar{x}_{0}, r\right)$;

(ii) $|F(\bar{y})| \leq \frac{1}{2}|F(\bar{x})|$.

Proof of (i): By Taylor's formula,

$$
\left|F\left(\bar{x}_{0}\right)-F(\bar{x})-F^{\prime}(\bar{x})\left[\bar{x}_{0}-\bar{x}\right]\right| \leq \sup \left|\frac{F^{\prime \prime}(\bar{z})}{2}\left[\bar{x}_{0}-\bar{x}, \bar{x}_{0}-\bar{x}\right]\right|,
$$

where $\bar{y} \in B\left(\bar{x}_{0}, r\right)$. Hence,

$$
\left|F\left(\bar{x}_{0}\right)-F(\bar{x})-F^{\prime}(\bar{x})\left[\bar{x}_{0}-\bar{x}\right]\right| \leq \frac{a_{2}}{2} n^{2}\left|\bar{x}_{0}-\bar{x}\right|^{2} .
$$

Now, using (1),

$$
\left|F\left(\bar{x}_{0}\right)-F^{\prime}(\bar{x})\left[\bar{x}_{0}-\bar{y}\right]\right| \leq \frac{a_{2}}{2} n^{2}\left|\bar{x}_{0}-\bar{x}\right|^{2} .
$$

Hence $\left|\bar{x}_{0}-\bar{y}\right| \leq\left|F^{\prime}(\bar{x})^{-1}\right|\left(\left|F\left(\bar{x}_{0}\right)\right|+\frac{a_{2}}{2} n^{2}\left|\bar{x}_{0}-\bar{x}\right|^{2}\right) \leq a_{0}\left(m+\frac{a_{2}}{2} n^{2} r^{2}\right) \leq r$ (the last inequality can be easily checked by substituting the values of $r, m$ ). 
Therefore $\bar{y} \in B\left(\bar{x}_{0}, r\right)$.

Proof of (ii): By Taylor's formula and using (1), we get

$$
|F(\bar{y})| \leq\left|F(\bar{x})+F^{\prime}(\bar{x})[\bar{y}-\bar{x}]\right|+\frac{a_{2}}{2} n^{2}|\bar{y}-\bar{x}|^{2}=0+\frac{a_{2}}{2} n^{2}|\bar{y}-\bar{x}|^{2}
$$

Another use of Taylor's Theorem yields

$$
|F(\bar{x})| \leq\left|F\left(\bar{x}_{0}\right)\right|+a_{1} n\left|\bar{x}-\bar{x}_{0}\right| .
$$

Hence, by (1),

$$
|\bar{y}-\bar{x}|^{2} \leq\left|F^{\prime}(\bar{x})^{-1}\right|^{2}|F(\bar{x})|^{2} \leq a_{0}^{2}\left(m+a_{1} n r\right)|F(\bar{x})| .
$$

Putting all together, $|F(\bar{y})| \leq \frac{a_{2}}{2} n^{2} a_{0}^{2}\left(m+a_{1} n r\right)|F(\bar{x})| \leq \frac{1}{2}|F(\bar{x})|$.

2.0.7 Theorem (Uniqueness Theorem for systems of linear differential equations). Let $a, b \in \mathbb{K} \cup\{ \pm \infty\}$ and $F=\left(f_{1}, \ldots, f_{n}\right):(a, b) \rightarrow \mathbb{K}^{n}$ a $C^{\infty}$ definable map. Let $A(t)=\left(a_{i j}(t)\right)$ be an $n \times n$ matrix of $C^{\infty}$ definable functions from $(a, b)$ to $\mathbb{K}$; suppose that

$$
\forall t \in(a, b) \quad F^{\prime}(t)=A(t) F(t) .
$$

Then, either $F$ is identically zero or else it never vanishes on $(a, b)$.

\section{Varieties of $C^{\infty}$ definable functions}

In this section we develop some differential topology for the class of topological $\mathbb{K}$-manifolds defined as zero-sets of definable smooth functions.

3.0.1 Definition. If $n, m \in \mathbb{N}$ and $U$ is a definable open subset of $\mathbb{K}^{n}$, let $C^{\infty}\left(U, \mathbb{K}^{m}\right)$ be the ring of $C^{\infty}$ definable maps from $U$ to $\mathbb{K}^{m}$.

If $G \in C^{\infty}\left(U, \mathbb{K}^{m}\right)$, let the variety of $G$ be the set $V(G)=\{\bar{a} \in U: G(\bar{a})=$ $\overline{0}\}$. If $G=\left(g_{1}, \ldots, g_{m}\right)$, then $V(G)=V\left(g_{1}\right) \cap \ldots \cap V\left(g_{m}\right)$; we will often write $V\left(g_{1}, \ldots, g_{m}\right)$ instead of $V(G)$.

3.0.2 Remark. The variety of $G$ is clearly a closed subset of $U$, for it is the preimage of a point under a continuous map.

3.0.3 Notation. If $n \geq m \in \mathbb{N}, G=\left(g_{1}, \ldots, g_{m}\right): \mathbb{K}^{n} \rightarrow \mathbb{K}^{m}$ is a $C^{\infty}$ definable map and $\bar{a} \in \mathbb{K}^{n}$, we denote by $D G(\bar{a})$ the $m \times n$ matrix corresponding to the linear map $G^{\prime}(a)$, with respect to the standard basis. The rows of $D G(\bar{a})$ are the vectors $\nabla g_{1}(\bar{a}), \ldots, \nabla g_{m}(\bar{a})$. If $\bar{y} \subset \bar{x}$ is a sub-tuple of coordinates, then we denote by $D_{\bar{y}} G(\bar{a})$ the matrix of the partial derivatives $\frac{\partial g_{i}}{\partial y_{j}}(\bar{a})$ with respect to the variables in the tuple $\bar{y}$. 
We will use many times in this work, some version of the Implicit Function Theorem. The statement is standard, but technical and we will find it useful to fix here a notation and to refer to this subsection whenever we use the theorem.

3.0.4 Definition. Let $n, m \in \mathbb{N}, U \subseteq \mathbb{K}^{n}$ open and definable and $G=$ $\left(g_{1}, \ldots, g_{m}\right) \in C^{\infty}\left(U, \mathbb{K}^{m}\right)$. Let $\bar{a} \in V(G)$ be a point such that the linear map $G^{\prime}(\bar{a})$ is surjective. Then we say that $\bar{a}$ is a regular point of $G$. The set of regular zeroes of $G$ (the regular set of $G$, for short) is denoted by $V^{\text {reg }}(G)$. So,

$$
V^{\mathrm{reg}}(G):=\left\{\bar{a} \in U: \bar{a} \in V(G) \text { and } G^{\prime}(\bar{a}) \text { is onto }\right\} .
$$

In other words, $V^{\mathrm{reg}}(G)$ is the set of those $\bar{a} \in V\left(g_{1}, \ldots, g_{m}\right)$ such that the vectors $\nabla g_{1}(\bar{a}), \ldots, \nabla g_{m}(\bar{a})$ are $\mathbb{K}$-linearly independent (We denote by lin. $\operatorname{span}\left\langle\nabla g_{1}(\bar{a}), \ldots, \nabla g_{m}(\bar{a})\right\rangle$ the $\mathbb{K}$-vector space generated by these vectors).

Notice that the definition of regular set depends crucially on the choice of $G$ : as a set, $V\left(g_{1}\right)=V\left(g_{1}^{2}\right)$; but $V^{\text {reg }}\left(g_{1}\right) \neq V^{\text {reg }}\left(g_{1}^{2}\right)$. In fact $V^{\text {reg }}\left(g_{1}^{2}\right)$ is always empty.

3.0.5 Remark. Using the result 3.0.7 proved below, we will see that $V^{\mathrm{reg}}(G)$ is locally definably diffeomorphic to an open subset of $\mathbb{K}^{n-m}$. Hence, $V^{\text {reg }}(G)$ is a differentiable $\mathbb{K}$-manifold, of dimension $n-m$.

We give now the notation which we will use for the Implicit Function Theorem and its Corollaries.

3.0.6 Notation. Let $n \geq m \in \mathbb{N}$. We write $n=k+m$ and we fix the following set of coordinates:

$$
\begin{aligned}
\mathbb{K}^{n} & =\mathbb{K}^{k} \times \mathbb{K}^{m} \\
\bar{x} & =(\bar{u}, \bar{v})
\end{aligned}
$$

Let $G=\left(g_{1}, \ldots, g_{m}\right) \in C^{\infty}\left(\mathbb{K}^{k+m}, \mathbb{K}^{m}\right)$ and $\bar{x}_{0}=\left(\bar{u}_{0}, \bar{v}_{0}\right) \in V(G)$ such that $D_{\bar{v}} G\left(\bar{x}_{0}\right)$ is non-singular.

3.0.7 Theorem (Implicit function Theorem). There exist

1. open definable subsets $O \subseteq \mathbb{K}^{k}$ and $W \subseteq \mathbb{K}^{m}$ such that $\bar{x}_{0} \in O \times W$, and

2. a definable $C^{\infty}$ map

$$
Y: O \rightarrow W
$$

such that $Y\left(\bar{u}_{0}\right)=\bar{v}_{0}$ and 


$$
\forall \bar{u} \in O \quad \forall \bar{v} \in W \quad G(\bar{u}, \bar{v})=\overline{0} \Leftrightarrow \bar{v}=Y(\bar{u}) .
$$

Moreover, $D_{\bar{u}} Y(\bar{u})$ is everywhere non-singular and, if $J_{\bar{v}} G(\bar{x})=$ $\operatorname{det} D_{\bar{v}} G(\bar{x})$,

$$
\forall \bar{x} \in U \quad D_{\bar{u}} Y(\bar{u})=-J_{\bar{v}} G^{-1}(\bar{u}, Y(\bar{u})) \cdot D_{\bar{u}} G(\bar{u}, Y(\bar{u})) .
$$

3.0.8 Definition. The map

$$
\begin{aligned}
\phi: \mathbb{K}^{k} & \rightarrow V(G) \cap O \times W \\
\bar{u} & \mapsto \quad(\bar{u}, Y(\bar{u}))
\end{aligned}
$$

is called a local rectangular parametrization of $V(G)$ around $\bar{x}_{0}$, and is a definable diffeomorphism, whose inverse is the restriction to $V(G) \cap(O \times W)$ of the projection $\pi: \mathbb{K}^{n} \rightarrow \mathbb{K}^{k}$ onto the first $k$ coordinates.

The proof of the Implicit Function Theorem works as in the o-minimal case (see for example [1]). The only nontrivial fact in this setting, which is used in the proof, is the following.

3.0.9 Theorem (Miller,[9]). Let $A \subseteq \mathbb{K}^{n}$ be closed, bounded and definable, and let $f: A \rightarrow \mathbb{K}^{m}$ be a continuous definable map. Then $f(A)$ is closed, bounded and definable.

We give now a list of the usual consequences of the Implicit Function Theorem.

3.0.10 Corollary. There is a ring homomorphism (the restriction homomorphism)

$$
\begin{aligned}
& \text { ^: } C^{\infty}(O \times W, \mathbb{K}) \rightarrow C^{\infty}(O, \mathbb{K}) \\
& h \quad \mapsto \widehat{h}(\bar{u})=h(\bar{u}, Y(\bar{u}))
\end{aligned}
$$

The kernel of ${ }^{-}$is the set $\left\{h \in C^{\infty}(O \times W, \mathbb{K}): h \uparrow V(G) \cap(O \times W) \equiv 0\right\}$, hence

$$
\widehat{C^{\infty}}(O \times W, \mathbb{K}) \cong C^{\infty}(V(G) \cap(O \times W), \mathbb{K}) .
$$

3.0.11 Corollary (Lagrange's Multipliers Rule). Let $h \in C^{\infty}(O \times W, \mathbb{K})$. A point $\bar{x}=(\bar{u}, Y(\bar{u})) \in V(G) \cap(O \times W)$ is a local extremum (maximum or minimum) of $h$ on $V(G)$ if and only if $\nabla \widehat{h}(\bar{u})=0$. Moreover,

$$
\nabla \widehat{h}(\bar{u})=0 \Leftrightarrow \nabla h(\bar{u}, Y(\bar{u})) \in \text { lin. } \operatorname{span}\left\langle\nabla g_{1}(\bar{u}, Y(\bar{u})), \ldots, \nabla g_{m}(\bar{u}, Y(\bar{u}))\right\rangle .
$$

3.0.12 Corollary. Suppose $M \subset C^{\infty}(U \times W, \mathbb{K})$ is a noetherian ring closed under differentiation. Then so is $\widehat{M}\left[\widehat{J_{\bar{v}} G^{-1}}\right]$.

Proof. Notice that $\widehat{M}\left[\widehat{J_{\bar{v}} G^{-1}}\right]$ is a finite extension of a homomorphic image of a noetherian ring, hence it is noetherian; moreover, an easy calculation and Corollary 3.0.10 show that $\widehat{M}\left[\widehat{J_{\bar{v}} G^{-1}}\right]$ is also closed under differentiation. 


\section{Noetherian differential rings}

4.0.1 Definition. Let $n \in \mathbb{N}$ and $U \subseteq \mathbb{K}^{n}$ be a definably connected definable open set. A ring $M$ with the following properties

- $M \subseteq C^{\infty}(U, \mathbb{K})$;

- $M$ is noetherian;

- $M$ is closed under partial differentiation;

- $M \supseteq \mathbb{Z}\left[x_{1}, \ldots, x_{n}\right]$.

is called a noetherian differential ring.

4.0.2 Example. Let $\left\langle\mathbb{K},+,-, \cdot,<, g_{1}, . ., g_{l}, 0,1, \ldots\right\rangle$ be a definably complete structure such that $g_{1}, . ., g_{l} \in C^{\infty}\left(\mathbb{K}^{n}, \mathbb{K}\right)$ satisfy a system of differential equations, with polynomial coefficients:

$$
\frac{\partial g_{i}}{\partial x_{j}}(\bar{x})=q_{i j}\left(\bar{x}, g_{1}, \ldots, g_{l}\right) \quad(i=1, \ldots, l ; j=1, \ldots, n),
$$

with $q_{i j} \in \mathbb{K}\left[\bar{x}, y_{1}, \ldots, y_{l}\right]$.

Examples of such structures over the real numbers are: $\overline{\mathbb{R}}:=\langle\mathbb{R},+,-, \cdot,<$ $, 0,1, \ldots\rangle,\langle\overline{\mathbb{R}}, \exp \rangle,\langle\overline{\mathbb{R}}, \tan \rangle,\langle\overline{\mathbb{R}}, \sin , \cos \rangle$ (notice that this last is a non ominimal example).

Then,

$$
\mathbb{F}\left[x_{1}, \ldots, x_{n}, g_{1}, \ldots, g_{l}\right],
$$

where $\mathbb{F}$ is a subfield of $\mathbb{K}$, is a noetherian differential subring of $C^{\infty}\left(\mathbb{K}^{n}, \mathbb{K}\right)$.

4.0.3 Remark. In the given examples, if $\mathbb{K}$ is a structure based on $\mathbb{R}$, then the functions $g_{1}, \ldots, g_{l}$ are not only $C^{\infty}$, but even analytic (by CauchyKowalesky Theorem, see for example [5]). On the other hand, if $M$ is a noetherian differential ring which is not a finitely generated algebra, then it does not necessarily follow that $M$ consists of real analytic functions. An non-analytic example will be exhibited in Section 7 .

We now fix a noetherian differential ring $M \subseteq C^{\infty}(U, \mathbb{K})$ in our definably complete structure $\mathbb{K}$, and we study the properties of the zero-sets of functions in $M$, using the approach suggested in [15]. The following result shows that the functions in $M$ have a "quasi-analytic" behaviour.

4.0.4 Lemma (Lack of flat functions). Let $I \subseteq M$ be an ideal closed under differentiation; then either $V(I)=\emptyset$ or $V(I)=U$. 
Proof. Since $M$ is noetherian, $I$ is finitely generated, say $I=\left\langle g_{1}, \ldots, g_{s}\right\rangle$, and hence $V(I)=V\left(g_{1}, . ., g_{s}\right)$ is a closed definable subset. If $V(I) \neq \emptyset$, since $U$ is definably connected, all we need to show is that $V(I)$ is open.

Suppose for a contradiction that this is not the case. Then there exists $\bar{x} \in V(I)$ which is not an interior point, i.e. given an arbitrary open box neighbourhood $B$ of $\bar{x}_{0}$, there exists a point $\bar{y}_{0} \in B$ which is not in $V(I)$. Without loss of generality, we may assume that $\bar{x}_{0}, \bar{y}_{0}$ differ in exactly one coordinate, say, the first one: $\bar{x}_{0}=\left(s, p_{2}, \ldots, p_{n}\right), \bar{y}_{0}=\left(t, p_{2}, \ldots, p_{n}\right)$ and $s \neq t$.

Recall that $\left\{g_{1}, \ldots, g_{s}\right\}$ is a set of generators for $I$. Since $I$ is closed under differentiation, it follows in particular that the derivatives with respect to the first coordinate $\partial g_{1} / \partial x_{1}(\bar{x}), \ldots, \partial g_{s} / \partial x_{1}(\bar{x})$ all belong to $I$, hence there exist functions $a_{i j}(\bar{x}) \in M$ such that

$$
\forall \bar{x}, \forall i=1, \ldots, s \quad \frac{\partial g_{i}}{\partial x_{1}}(\bar{x})=\sum_{j=1}^{s} a_{i j}(\bar{x}) g_{j}(\bar{x}) .
$$

Now, consider the restrictions $f_{i}\left(x_{1}\right)=g_{i}\left(x_{1}, p_{2}, \ldots, p_{n}\right)$ of the functions $g_{1}, \ldots, g_{s}$ to the line $L=\left\{\bar{x} \in U: x_{2}=p_{2} \wedge \ldots \wedge x_{n}=p_{n}\right\}$, and define $F\left(x_{1}\right)=\left(f_{1}\left(x_{1}\right), \ldots, f_{s}\left(x_{1}\right)\right)$. We have

$$
F^{\prime}\left(x_{1}\right)=A\left(x_{1}\right) F\left(x_{1}\right)
$$

where $A\left(x_{1}\right)$ is the $s \times s$ matrix whose entries are the functions $a_{i j}\left(x_{1}, p_{2}, \ldots, p_{n}\right)$.

It follows from the Uniqueness Theorem for Linear Differential Equations 2.0.7 that either $F \equiv 0$ or else has no zeros. But this leads to a contradiction, since $F(t) \neq 0$ and $F(s)=0$.

4.0.5 Corollary. Let $G=\left(g_{1}, \ldots, g_{m}\right) \in M^{m}$ and $\bar{x}_{0} \in V^{\mathrm{reg}}(G)$. Then either there exists $h \in M$ such that $\bar{x}_{0} \in V^{\mathrm{reg}}(G, h)$ or for all $h \in M$, if $h\left(\bar{x}_{0}\right)=0$, then $h$ vanishes on a definable neighbourhood of $\bar{x}_{0}$ in $V^{\mathrm{reg}}(G)$.

Proof. We refer to the notation of the Implicit Function Theorem 3.0.7, so $\bar{x}_{0}=\left(\bar{u}_{0}, \bar{v}_{0}\right) \in \mathbb{K}^{k} \times \mathbb{K}^{m}$. Up to some rearrangement of the variables, we may assume that $D_{\bar{v}} G\left(\bar{x}_{0}\right)$ is non-singular and apply the Implicit Function Theorem in a suitable neighbourhood $O \times W$ of $\bar{x}_{0}$. Suppose that there is no $h \in M$ such that $\bar{x}_{0} \in V^{\mathrm{reg}}(G, h)$ and let $h \in M$ be such that $h\left(\bar{x}_{0}\right)=0$. Then $\nabla h\left(\bar{x}_{0}\right)$ belongs to lin. $\operatorname{span}\left\langle\nabla g_{1}\left(\bar{x}_{0}\right), \ldots, \nabla g_{m}\left(\bar{x}_{0}\right)\right\rangle$. This implies, by Lagrange's Multiplier Rule 3.0.11, that $\nabla \widehat{h}\left(\bar{u}_{0}\right)=0$.

Consider the ideal $\widehat{I}=\left\{\widehat{g} \in \widehat{M}\left[\widehat{J_{\bar{v}} G^{-1}}\right]: \widehat{g}(\bar{u})=0\right\}$; what we have shown is that if $\widehat{h} \in \widehat{I}$, then its first derivatives $\partial \widehat{h} / \partial u_{i}$ belong to $\widehat{I}$; thus $\widehat{I}$ 
is closed under differentiation. Since $V(\widehat{I}) \neq \emptyset$, it follows from Lemma 4.0.4 and the subsequent Remark, that $V(\widehat{I})=O$. This means that $h$ vanishes on $V^{\mathrm{reg}}(G) \cap(O \times W)$.

\section{Decomposition of noetherian varieties}

We fix, for the rest of the section, a noetherian differential ring $M$. The zero-sets of functions belonging to $M$ are called $M$-varieties. We prove the main result of this paper, namely that every $M$-variety can be decomposed into finitely many differentiable $\mathbb{K}$-manifolds of a certain form.

5.0.1 Definition. Let $A$ be a definable set; we say that $S$ is a definable clopen of $A$ if $S \subseteq A$ is a definable subset which is both open and closed in $A$. Clearly, the collection of all definable clopen of $A$ is a boolean algebra $\mathcal{B}(A)$ of sets.

5.0.2 Definition. If $G \in M^{m}$ and $S$ is a clopen definable subset of $V^{\mathrm{reg}}(G)$, then $S$ is called a regular component. The dimension of $S$ is the $\mathbb{K}$-manifold dimension of $V^{\mathrm{reg}}(G)$, which is $n-m$.

5.0.3 Lemma. Let $0 \neq f \in M$ and $V(f) \subset \mathbb{K}^{n}$ be a nonempty $M$-variety; then for all $\bar{x} \in V(f)$ there exists $g \in M$ such that $\bar{x} \in V^{\text {reg }}(g)$, i.e. $g(\bar{x})=$ $0 \wedge \nabla g(\bar{x}) \neq \overline{0}$

Proof. Take $\bar{x} \in V(f)$ and consider $f$ together with all its partial derivatives, evaluated in $\bar{x}$. We claim that there exist a multi-index $\alpha=\left(\alpha_{1}, \ldots, \alpha_{n}\right)$ and $i_{0} \in\{1, \ldots, n\}$ such that, if we put $\partial^{\alpha} f:=\frac{\partial^{\alpha_{1}+\ldots+\alpha_{n}}}{\partial x_{1}^{\alpha_{1} \ldots \ldots} x_{n}^{\alpha_{n}}}$, then $\partial^{\alpha} f(\bar{x})=0$ and $\frac{\partial \partial^{\alpha} f}{\partial x_{i_{0}}}(\bar{x}) \neq 0$, so that we can define $g:=\partial^{\alpha} f$. Suppose, on the contrary, that $f$ as well as all its derivatives $\partial^{\alpha} f$ vanishes in $\bar{x}$ and let $I$ be the ideal of $M$ generated by $f$ and all its derivatives. Notice that $V(I) \neq \emptyset$, since $\bar{x} \in V(I) ; M$ is noetherian, so $I$ is finitely generated. Moreover, $I$ is closed under differentiation, since each member of $I$ can be written as a linear combination (with coefficients in $M$ ) of a finite number of derivatives of $f$. Then, Lemma 4.0.4 implies that $V(I)$ (and hence $V(f)$ ) coincides with $\mathbb{K}^{n}$, which is impossible.

5.0.4 Remark. The above argument shows also that, if $0 \neq f \in M$, then $V(f)$ has empty interior. In fact, if $V(f)$ has interior around a point $\bar{x}$, then $\bar{x}$ is necessarily not a regular zero of $f$ (otherwise, by the Implicit Function Theorem 3.0.7, $V(f)$ would be locally diffeomorphic to $\mathbb{K}^{n-1}$ around $\bar{x}$ ). For the same reason, $\bar{x}$ is not a regular zero of any of the derivatives of $f$, hence 
all the derivatives of any order of $f$ vanish in $\bar{x}$. But then, as in the proof above, $V(f)$ must be $\mathbb{K}^{n}$.

5.0.5 Definition. For every $\bar{x} \in \mathbb{K}^{n}$, we define the $M$-degree of $\bar{x}, \operatorname{deg}_{M}(\bar{x})$, as the minimal dimension of a regular component containing $\bar{x}$. Equivalently,

$$
\operatorname{deg}_{M}(\bar{x})=\min \left\{k \mid \exists G \in M^{n-k} \text { such that } \bar{x} \in V^{\text {reg }}(G)\right\} \text {. }
$$

Lemma 5.0.3 shows that every point belonging to a proper $M$-variety has $M$-degree at most $n-1$.

5.0.6 Theorem. Let $f \in M$ and $V(f)$ be a proper $M$-variety. Then, for every point $\bar{x}$ in $V(f)$, there exist $k<n$ and $G \in M^{n-k}$ and a regular component $S$ of $V^{\mathrm{reg}}(G)$ such that $\bar{x} \in S \subseteq V(f)$. Moreover, $S$ is definable with the same parameters used to define $G$ and $f$.

Proof. Let $k=\operatorname{deg}_{M}(\bar{x})$ and $G \in M^{n-k}$ such that $\bar{x} \in V^{\text {reg }}(G)$. We define

$$
S:=\text { the interior of the set } V^{\mathrm{reg}}(G) \cap V(f) \text { in } V^{\mathrm{reg}}(G) \text {. }
$$

We claim that $\bar{x} \in S$. In fact, by the choice of $V^{\text {reg }}(G)$ as a regular set of minimal dimension, by Corollary 4.0.5 it follows that every function $h \in M$ which vanishes in $\bar{x}$, also vanishes on an open definable neighbourhood $B$ of $\bar{x}$ in $V^{\mathrm{reg}}(G)$. In particular, $f$ vanishes on some $B$ (depending on $f$ ). Hence $\bar{x}$ has an open neighbourhood $B$ contained in $V(f) \cap V^{\mathrm{reg}}(G)$, i.e. $\bar{x}$ is an interior point.

We now claim that $S$ is a regular component. $S$ is definable, nonempty and open in $V^{\operatorname{reg}}(G)$, by definition. We must show that $S$ is also closed in $V^{\mathrm{reg}}(G)$. Take a boundary point $\bar{x}_{0}$ of $S$ in $V^{\mathrm{reg}}(G)$ and consider (after permuting the variables, if necessary) the local parametrization given by the Implicit Function Theorem 3.0.7

$$
\begin{array}{cccc}
\phi: \mathbb{K}^{k} & \rightarrow & V(G) \cap(O \times W) \\
\bar{u} & \mapsto & (\bar{u}, Y(\bar{u})) .
\end{array}
$$

Setting, as usual, $\widehat{f}=f \circ \phi$, we observe that $\phi^{-1}(S)$ is open in $O$ and $\widehat{f}\left(\phi^{-1}(S)\right)=0$. Hence, all derivatives of any order of $\widehat{f}$ vanish on $\phi^{-1}(S)$. Since $\bar{u}_{0}=\phi^{-1}\left(\bar{x}_{0}\right)$ belongs to the closure of $\phi^{-1}(S)$, it is also true, by continuity, that $\widehat{f}$, and all its derivatives of any order, vanish in $\bar{u}_{0}$. By Lemma 4.0.4 and the usual argument, $V(\widehat{f})=\mathbb{K}^{k}$. Hence, the open neighbourhood $O \times W$ of $\bar{x}_{0}$ is contained in $V(f) \cap V^{\mathrm{reg}}(G)$, that implies $\bar{x}_{0} \in S$. 
5.0.7 Theorem (Decomposition of $M$-varieties). Let $f \in M$ and $V(f)$ be a proper $M$-variety. Then $V(f)$ can be written as a finite union of regular components:

$\exists k \in \mathbb{N}, \exists G_{1}, \ldots, G_{k} \in \bigcup_{l=1}^{n} M^{l}, \exists S_{i} \in \mathcal{B}\left(V^{\mathrm{reg}}\left(G_{i}\right)\right)$ so that $V(f)=S_{1} \cup \ldots \cup S_{k}$.

Proof. By compactness. More precisely, let $\mathbb{F}$ be a $|\mathbb{K}|^{+}$-saturated elementary superstructure of $\mathbb{K}$ (see [13] for the existence of such an $\mathbb{F}$ ), so that $\mathbb{F}$ realizes all types over $\mathbb{K}$. Let $\tilde{M}$ be the set of those definable functions $\tilde{g}$ such that $g \in M$ and $\tilde{g}$ is the interpretation of $g$ in $\mathbb{F}$ (note that $\tilde{g}$ is still a $C^{\infty}$ function). Then $\tilde{M}$ is still a noetherian differential ring, hence Theorem 5.0.6 holds for $\tilde{M}$-varieties. Consider the function $\tilde{f}$ and the following set of formulas:

$\Phi=\left\{\phi_{\tilde{G}}:=\bar{x} \in V(\tilde{f}) \wedge \tilde{S}=\operatorname{int}_{V^{\operatorname{reg}(\tilde{G})}}\left(V^{\operatorname{reg}}(\tilde{G} \cap V(\tilde{f})) \wedge(\bar{x} \in \tilde{S} \rightarrow \tilde{S} \not \subset V(\tilde{f})) \mid \tilde{G} \in \bigcup_{i=1}^{n} \tilde{M}^{i}\right\}\right.$

If $\Phi$ were a consistent type, then it would be realized $\mathbb{F}$. This means that there would exists an $\bar{x} \in \mathbb{F}$ such that for all $\tilde{G} \in \bigcup_{i=1}^{n} \tilde{M}^{i}, \bar{x} \in V(\tilde{f}) \wedge \tilde{S}=$ $\operatorname{int}_{V^{\mathrm{reg}}(\tilde{G})}\left(V^{\mathrm{reg}}(\tilde{G} \cap V(\tilde{f})) \wedge(\bar{x} \in \tilde{S} \rightarrow \tilde{S} \not \subset V(\tilde{f}))\right.$, which would contradict Theorem 5.0.6. Hence there exist $k \in \mathbb{N}, \tilde{G}_{1}, \ldots, \tilde{G}_{k} \in \bigcup_{i=1}^{n} \tilde{M}^{i}$, such that the conjunction $\phi_{\tilde{G}_{1}} \wedge \ldots \wedge \phi_{\tilde{G}_{k}}$ is not satisfiable in $\mathbb{F}$; in other words the following holds in $\mathbb{F}$ :

$$
\forall \bar{x}\left(\bar{x} \in \tilde{S}_{1} \cup \ldots \cup \tilde{S}_{k} \wedge \tilde{S}_{1} \cup \ldots \cup \tilde{S}_{k} \subseteq V(\tilde{f})\right) .
$$

Therefore $V(\tilde{f})=\tilde{S}_{1} \cup \ldots \cup \tilde{S}_{k}$.

Now, in $\mathbb{K}$ the following holds: $V(f)=S_{1} \cup \ldots \cup S_{k}$, where $S_{i}:=\tilde{S}_{i} \cap \mathbb{K}^{n}$ $(i=1, \ldots, k)$ are clearly regular components in $\mathbb{K}$, hence the theorem is proved.

5.0.8 Remark. The decomposition which appears in Theorem 5.0.7 is clearly not unique, nor are unique the dimensions of the regular components appearing in two different decompositions of the same variety. For example, the algebraic variety $V\left(x^{2}-y^{2}\right) \subset \mathbb{R}^{2}$ can be decomposed as $V^{\text {reg }}\left(x^{2}-y^{2}\right) \cup V^{\text {reg }}(x, y)$ or as $V^{\text {reg }}(x-y) \cup V^{\text {reg }}(x+y)$. In the first decomposition the first regular component has dimension 1 and the second has dimension 0 , while in the second decomposition both regular components have dimension 1. Moreover, in the first case the union is disjoint, and in the second case it is not.

On the other hand, the following holds: 
5.0.9 Lemma. Let $f \in M$. Then there exists a unique natural number $m$ such that for every decomposition of $V(f)$ into regular components, as in Theorem 5.0.7, the maximal dimension of the regular components appearing in the decomposition is $m$.

Proof. Let $V(f)=S_{1} \cup \ldots \cup S_{k}$ be a decomposition of $V(f)$ into regular components and suppose $\operatorname{dim} S_{i} \leq \operatorname{dim} S_{1}=m$, for all $i=2, \ldots, k$. Clearly $V(f)$ does not contain an open subset which is diffeomorphic to $\mathbb{K}^{l}$, for $l>m$, because otherwise such a subset would be obtained as a finite union of manifolds of dimension $\leq m$, which, as in the classical case, is not possible. On the other hand, $V(f)$ does contain an open subset which is diffeomorphic to $\mathbb{K}^{m}$, because so does $S_{1}$. Hence, every decomposition of $V(f)$ must contain a component of dimension $m$, and can not contain components of bigger dimension.

5.0.10 Definition. The dimension of an $M$-variety $V(f)$ is defined as

$$
\operatorname{dim} V(f):=\max \left\{\operatorname{dim} S_{i} \mid i=1, \ldots, k \text { and } V(f)=S_{1} \cup \ldots \cup S_{k}\right\},
$$

where $V(f)=S_{1} \cup \ldots \cup S_{k}$ is any decomposition given by Theorem 5.0.7.

5.0.11 Lemma. Let $g_{1}, \ldots, g_{m} \in M$ and $\bar{x} \in V^{\mathrm{reg}}\left(g_{1}, \ldots, g_{m}\right) \subset \mathbb{K}^{n}$. If $\operatorname{deg}_{M}(\bar{x})<n-m$, then there exists $f \in M$ such that $\bar{x} \in V^{\mathrm{reg}}\left(g_{1}, \ldots, g_{m}, f\right)$.

Proof. Since $\operatorname{deg}_{M}(\bar{x})<n-m$, there exist $f_{1}, \ldots, f_{m+1} \in M$ so that $\bar{x} \in V^{\mathrm{reg}}\left(f_{1}, \ldots, f_{m+1}\right)$. We claim that there exists $i \in\{1, \ldots, m+1\}$ so that $\bar{x} \in V^{\text {reg }}\left(g_{1}, \ldots, g_{m}, f_{i}\right)$, because otherwise the (linearly independent) vectors $\nabla f_{1}(\bar{x}), \ldots, \nabla f_{m+1}(\bar{x})$ would all lie in the $m$-dimensional vector space generated by $\nabla g_{1}(\bar{x}), \ldots, \nabla g_{m}(\bar{x})$, which is impossible.

5.0.12 Proposition. Let $V(f) \subset \mathbb{K}^{n}$ be an $M$-variety. Let $\mathbb{F}$ be any $|\mathbb{K}|^{+}$-saturated elementary superstructure of $\mathbb{K}$ and let $\tilde{f}$ be the interpretation of $f$ in $\mathbb{F}$ (as in the proof of 5.0.7). Then,

$$
\operatorname{dim} V(f)=\max \left\{\operatorname{deg}_{M}(\bar{x}) \mid \bar{x} \in V(\tilde{f})\right\} .
$$

Proof. Let $V(\tilde{f})=\tilde{S}_{1} \cup \ldots \cup \tilde{S}_{l}$ be a decomposition of $V(\tilde{f})$ into regular components, and let $S_{i}=\mathbb{K}^{n} \cap \tilde{S}_{i}$. Then $V(f)=S_{1} \cup \ldots \cup S_{l}$, hence $\operatorname{dim} V(\tilde{f})=\operatorname{dim} V(f)$. Let $\bar{x} \in V(\tilde{f})$. Then $\bar{x} \in \tilde{S}_{i}$ for some $i$, hence $\operatorname{deg}_{M}(\bar{x}) \leq \operatorname{dim} \tilde{S}_{i} \leq \operatorname{dim} V(f)$. So $\operatorname{dim} V(f) \geq \max \left\{\operatorname{deg}_{M}(\bar{x}) \mid \bar{x} \in V(\tilde{f})\right\}$.

Now we prove that there exists $\bar{x} \in V(\tilde{f})$ with $\operatorname{deg}_{M}(\bar{x})=\operatorname{dim} V(f)$. Let $\tilde{S}_{1} \in \mathcal{B}\left(V^{\mathrm{reg}}\left(\tilde{g_{1}}, \ldots, \tilde{g}_{k}\right)\right)$ be a component of maximal dimension. Consider the set of formulas

$$
\Phi=\left\{\bar{x} \in \tilde{S}_{1} \wedge \bar{x} \notin V^{\mathrm{reg}}\left(\tilde{g_{1}}, \ldots, \tilde{g_{k}}, \tilde{h}\right) \mid h \in M\right\} .
$$


$\Phi$ is clearly finitely satisfiable in $\mathbb{F}$, because no finite union of regular sets of dimension $n-k-1$ can cover the whole of $\tilde{S}_{1}$, which has dimension $n-k$. By saturation, there exists $\bar{x} \in \mathbb{F}^{n}$ which satisfies all formulas in $\Phi$. By Lemma 5.0.11, then, $\operatorname{deg}_{M}(\bar{x})=\operatorname{dim} V(f)$.

\section{Khovanskii rings}

In this section we give an application of the Decomposition Theorem 5.0.7. We consider a class of noetherian differential rings, called Khovanskii rings, with the property that 0-dimensional regular sets are finite. An example of such a ring is $M=\mathbb{R}\left[\bar{x}, f_{1}, \ldots, f_{k}\right]$, where the functions $f_{i}$ form a Pfaffian chain, as proved by Khovanskii in [6]. In the same paper, it is proven that all $M$-varieties, where $M$ is as above, have finitely many connected components. Here we prove, with a method which differs from the approach in [6], that if $M$ is a Khovanskii ring in a definably complete structure, then all $M$-varieties have finitely many definably connected components.

This result can be compared with Theorem 1.7 in [3], which is formulated in the setting of Rolle leaves.

6.0.1 Definition (Khovanskii rings). Let $n \in \mathbb{N}$. A ring $M$ with the following properties

1. $M \subseteq C^{\infty}\left(\mathbb{K}^{n}, \mathbb{K}\right)$

2. $M$ is a noetherian differential ring;

3. $\forall g_{1}, \ldots, g_{n} \in M \quad\left|V^{\mathrm{reg}}\left(g_{1}, \ldots, g_{n}\right)\right|<\infty$.

is called a Khovanskii ring.

A collection of rings $\left\{M_{n} \mid n \in \mathbb{N}\right\}$ such that

1. $M_{n}$ is a ring of definable $C^{\infty}$ functions from $\mathbb{K}^{n}$ to $\mathbb{K}$;

2. $M_{n}$ is a Khovanskii ring;

3. $M_{n} \subset M_{n+1}$ (in the obvious sense);

4. $M_{n}$ is closed under permutation of the variables.

is called a collection of Khovanskii rings.

A similar definition appears in [2].

6.0.2 Examples. Examples of Khovanskii rings over the real numbers are: 
- The ring generated by a Pfaffian chain of functions (see [6]);

- Any noetherian differential ring of functions definable in an o-minimal expansion of the real field;

- The ring generated by the real functions $\exp \left(\frac{1}{1+x^{2}}\right), \sin \left(\frac{1}{1+x^{2}}\right)$ and $\cos \left(\frac{1}{1+x^{2}}\right)($ see $[7])$

6.0.3 Remark. Fix $n, m \in \mathbb{N}, m \leq n$. Let $M \subseteq C^{\infty}\left(\mathbb{K}^{n}, \mathbb{K}\right)$ be a noetherian differential ring (not necessarily a Khovanskii ring) and let $F \in M^{m}$. Then the set of regular zeroes of $F$ can be expressed as the projection of a finite union of regular varieties of $\operatorname{dimension} \operatorname{dim} V^{\mathrm{reg}}(F)$. To see this, let $E_{1}(\bar{x}), \ldots, E_{l}(\bar{x})$ be the maximum rank minors of the matrix $D F(\bar{x})$. Now consider $V_{i}:=V\left(F(\bar{x}), x_{n+1} \operatorname{det} E_{i}(\bar{x})-1\right)$. Then $V_{i}$ is a regular sub-variety of $\mathbb{K}^{n+1}$ and $\pi_{n+1}\left(\bigcup_{i=1}^{l} V_{i}\right)=V^{\mathrm{reg}}(F)$ (where $\pi_{n+1}$ is the projection onto the first $n$ coordinates).

Notice that $\operatorname{dim} V_{i}=n+1-(m+1)=n-m=\operatorname{dim} V^{\mathrm{reg}}(F)$. Moreover, if $M=M_{n}$ belongs to a collection of Khovanskii rings, then the map $\left(F(\bar{x}), x_{n+1} \operatorname{det} E_{i}(\bar{x})-1\right)$ belongs to $M_{n+1}^{n-k+1}$.

6.0.4 Proposition. Fix $n, m \in \mathbb{N}, m \leq n-1$. Let $M \subseteq C^{\infty}\left(\mathbb{K}^{n}, \mathbb{K}\right)$ be a Khovanskii ring and $f_{1}, \ldots, f_{m} \in M$ be such that $V\left(f_{1}, \ldots, f_{m}\right)=$ $V^{\mathrm{reg}}\left(f_{1}, \ldots, f_{m}\right)$. Then there exists a definable set $G$ such that:

- $\emptyset \neq G \subset V\left(f_{1}, \ldots, f_{m}\right)$;

- For every clopen definable subset $S$ of $V\left(f_{1}, \ldots, f_{m}\right)$, the intersection $S \cap G$ is not empty;

- $\forall \bar{x} \in G \exists h \in M \quad\left(\bar{x} \in V^{\mathrm{reg}}\left(f_{1}, \ldots, f_{m}, h\right)\right)$.

Proof. For all $h \in M$, consider the matrix of partial derivatives $D\left(f_{1}, \ldots, f_{m}, h\right)$. Let $\bar{x} \in V\left(f_{1}, \ldots, f_{m}\right)$. Then, this matrix, if we evaluate all the entries in $\bar{x}$, has rank at least $m$, because the common zeroes of the functions $f_{1}, \ldots, f_{m}$ are all regular zeroes, by hypothesis. Let $H_{i}(\bar{x})$ $\left(i=1, \ldots,\left(\begin{array}{c}n \\ m+1\end{array}\right)\right)$ be the minors of rank $m+1$ of the matrix $D\left(f_{1}, \ldots, f_{m}, h\right)$ evaluated in $\bar{x}$ and define $h^{*}(\bar{x})=\sum_{i=1}^{l}\left(\operatorname{det} H_{i}\right)(\bar{x})^{2} \in M$. Then $\bar{x}$ is a critical point of $h$ on $V\left(f_{1}, \ldots, f_{m}\right)$ if and only if $h^{*}(\bar{x})=0$. And (see 3.0.11),

$$
h^{*}(\bar{x})=0 \Leftrightarrow \nabla h(\bar{x}) \in \operatorname{lin} . \operatorname{span}\left(\nabla f_{1}(\bar{x}), \ldots, \nabla f_{m}(\bar{x})\right) .
$$

We take $n+1$ points $P_{0}, \ldots, P_{n}$ in $\mathbb{Z}^{n}$ such that the vectors $\overrightarrow{P_{0}} P_{1}, \ldots, \overrightarrow{P_{0} P_{n}}$ are linearly independent over $\mathbb{K}$. For example, let us take $P_{0}=\overline{0}$ and $P_{i}$ to be 
the tuple with the $i$-th coordinate equal to 1 and the other coordinates equal to 0 (for $i=1, \ldots, n$ ). Now consider the following "distance" functions:

$$
d_{0}(\bar{x})=\sum_{j=1}^{n} x_{j}^{2}, \quad d_{i}(\bar{x})=\left(x_{i}-1\right)^{2}+\sum_{j=1, j \neq i}^{n} x_{j}^{2} \quad i=1, \ldots, n .
$$

Clearly these functions belong to $M$.

For every $S \in \mathcal{B}\left(V\left(f_{1}, \ldots, f_{m}\right)\right)$, for every $i=0, \ldots, n$, consider the set $V_{S}\left(d_{i}^{*}\right)=S \cap V\left(d_{i}^{*}\right)$ of the critical points of the function $d_{i}$ on $S$ and let $\operatorname{bd}_{S} V_{S}\left(d_{i}^{*}\right)=V_{S}\left(d_{i}^{*}\right) \backslash \operatorname{int}_{S}\left(V_{S}\left(d_{i}^{*}\right)\right)$ be the set of boundary points of $V_{S}\left(d_{i}^{*}\right)$ in $S$. Now define

$$
G:=\bigcup_{S \in \mathcal{B}\left(V\left(f_{1}, \ldots, f_{m}\right)\right)} \bigcup_{i=0, \ldots, n} \operatorname{bd}_{S} V_{S}\left(d_{i}^{*}\right)
$$

Step 1. We first observe that $G$ is definable and $G \subseteq V\left(f_{1}, \ldots, f_{m}\right)$.

Step 2. Next, we note that for all $S \in \mathcal{B}\left(V\left(f_{1}, \ldots, f_{m}\right)\right)$, for every $i=0, \ldots, n$, the set $S$ contains a point whose distance from $P_{i}$ is minimal, i.e. $V_{S}\left(d_{i}^{*}\right)$ is nonempty. This follows from the fact that $d_{i}$ increases on balls centered in $P_{i}$ and of increasing radius, so Theorem 2.0.3 applies.

Step 3. Now we show that $G$ meets every nonempty definable clopen of $V\left(f_{1}, \ldots, f_{m}\right)$ (in particular, $G$ is not empty). Equivalently, we show that for all $S \in \mathcal{B}\left(V\left(f_{1}, \ldots, f_{m}\right)\right) \backslash\{\emptyset\}$, there exists $i \in\{0, \ldots, n\}$ such that the set $V_{S}\left(d_{i}^{*}\right)$ is not open in $S$. Suppose for a contradiction that this is not the case. Then for all $i=0, \ldots, n$ the set $V_{S}\left(d_{i}^{*}\right)$, which is clearly closed and definable, in also open in $V\left(f_{1}, \ldots, f_{m}\right)$, and hence it belongs to $\mathcal{B}\left(V\left(f_{1}, \ldots, f_{m}\right)\right)$. Now consider the boolean subalgebra $\mathcal{A}$ of $\mathcal{B}\left(V\left(f_{1}, \ldots, f_{m}\right)\right)$ generated by $V_{S}\left(d_{0}^{*}\right), \ldots, V_{S}\left(d_{n}^{*}\right)$. Since $\mathcal{A}$ is finite, there is an atom, say, $C \in \mathcal{A}$. Let $C_{i}=C \cap V_{S}\left(d_{i}^{*}\right)$; by Step $1, C_{i}$ is nonempty for all $i=0, \ldots, n$, and hence $C_{i}=C$. But this implies that $\emptyset \neq C \subseteq V\left(d_{0}^{*}, \ldots, d_{n}^{*}\right)$. But this is not possible, because the vectors $\nabla d_{i}(\bar{x})$ span $\mathbb{K}^{n}$ at all points $\bar{x}$. If $\bar{x} \in V\left(d_{0}^{*}, \ldots, d_{n}^{*}\right)$, then $\forall i=0, \ldots, n, \nabla d_{i}(\bar{x}) \in$ lin. $\operatorname{span}\left(\nabla f_{1}(\bar{x}), \ldots, \nabla f_{m}(\bar{x})\right)$, which is absurd.

Step 4. We now show that $\forall \bar{x} \in G \exists h \in M \quad \bar{x} \in V^{\mathrm{reg}}\left(f_{1}, \ldots, f_{m}, h\right)$. Suppose for a contradiction that there exists $\bar{x} \in G$ such that it is not possible to cut transversally $V\left(f_{1}, \ldots, f_{m}\right)$ at $\bar{x}$ by any $h \in M$. Now arguing as in the last paragraph of the proof of Theorem 5.0.6, we show that every $h \in M$ must vanish on a suitable neighbourhood of $\bar{x}$ in $V\left(f_{1}, \ldots, f_{m}\right)$. But by definition of $G$, every point $\bar{x}$ of $G$ is a boundary point of some $V_{S}\left(d_{i}^{*}\right)$, i.e.

$$
\begin{aligned}
& \forall \bar{x} \in G \quad \exists S \in \mathcal{B}\left(V\left(f_{1}, \ldots, f_{m}\right)\right) \quad \exists i \in\{0, \ldots, s\} \\
& d_{i}^{*}(\bar{x})=0 \quad \wedge \forall r>0 \exists \bar{y} \in S \cap B(\bar{x}, r) d_{i}^{*}(\bar{y}) \neq 0,
\end{aligned}
$$

and this leads to a contradiction. 
6.0.5 Theorem. Fix $n, m \in \mathbb{N}, m \leq n-1$. Let $M \subseteq C^{\infty}\left(\mathbb{K}^{n}, \mathbb{K}\right)$ be a Khovanskii ring and $F \in M^{m}$ be such that $V(F)=V^{\mathrm{reg}}(F)$. Then there exists a definable set $G$ such that:

- $\emptyset \neq G \subset V(F)$;

- For every clopen definable subset $S$ of $V(F)$, the intersection $S \cap G$ is not empty;

- $\exists l \in \mathbb{N}, \exists h_{1}, \ldots, h_{l} \in M \quad G \subset V^{\mathrm{reg}}\left(F, h_{1}\right) \cup \ldots \cup V^{\mathrm{reg}}\left(F, h_{l}\right)$.

Proof. By compactness, using an argument similar to the one used in the proof of 5.0.7. More precisely, let $\mathbb{F}$ be a $|\mathbb{K}|^{+}$-saturated elementary superstructure of $\mathbb{K}$ (see [13] for the existence of such an $\mathbb{F}$ ), so that $\mathbb{F}$ realizes all types over $\mathbb{K}$. Let $\widetilde{M}$ be the set of those definable functions $\tilde{g}$ such that $g \in M$ and $\tilde{g}$ is the interpretation of $g$ in $\mathbb{F}$ (note that $\tilde{g}$ is still a $C^{\infty}$ function). Then $\widetilde{M}$ is still a Khovanskii ring, hence Proposition 6.0.4 holds for $\widetilde{M}$-varieties. Consider the map $\widetilde{F}=\left(\widetilde{f}_{1}, \ldots, \widetilde{f}_{m}\right)$ and the following set of formulas:

$$
\Phi=\left\{\phi_{\widetilde{h}}:=\left(\bar{x} \in G \wedge \bar{x} \notin V^{\mathrm{reg}}(\widetilde{F}, \widetilde{h})\right) \mid h \in M\right\} .
$$

If $\Phi$ were a consistent type, then it would be realized in $\mathbb{F}$. This means that there would exist $\bar{x} \in G$ such that for all $h \in M, \bar{x} \notin V^{\mathrm{reg}}(\widetilde{F}, \widetilde{h})$, which is not possible by Proposition 6.0.4. Hence there exist $h_{1}, \ldots, h_{l} \in M$ such that the conjunction $\phi_{\widetilde{h}_{1}} \wedge \ldots \wedge \phi_{\widetilde{h}_{l}}$ is not satisfiable; in other words the following holds in $\mathbb{F}$ :

$$
\forall \bar{x} \bar{x} \in G \rightarrow \bar{x} \in V^{\mathrm{reg}}\left(\widetilde{F}, \widetilde{h}_{1}\right) \cup \ldots \cup V^{\mathrm{reg}}\left(\widetilde{F}, \widetilde{h}_{l}\right)
$$

Pulled back to $\mathbb{K}$, this proves the theorem.

6.0.6 Theorem (Finiteness of $\mathcal{B}(V(F))$ ). Let $\left\{M_{n} \mid n \in \mathbb{N}\right\}$ be a collection of Khovanskii rings. Then, for all $n, m \in \mathbb{N}$ and $F \in\left(M_{n}\right)^{m}$, the boolean algebra $\mathcal{B}(V(F))$ is finite.

Proof. By induction, using Propositions 6.0.5, 5.0.7 and Remark 6.0.3.

More precisely, we first prove by induction on $k=n-m$ that $\forall n \in \mathbb{N}, \mathcal{B}\left(V^{\text {reg }}(F)\right)$ is finite.

The case $k=0$ follows from the fact that $M_{n}$ is a Khovanskii ring. Next, suppose the statement true for $n-m<k$ and consider $F \in M_{n}^{n-k}$. If $V(F)=$ $V^{\text {reg }}(F)$, then there exist a definable set $G$ and functions $h_{1}, \ldots, h_{l} \in M_{n}$ as in Theorem 6.0.5.

By inductive hypothesis, $\mathcal{B}\left(V^{\mathrm{reg}}\left(F, h_{i}\right)\right)$ is finite, and hence so is $\mathcal{B}\left(V^{\mathrm{reg}}(F)\right)$, assuming $V(F)=V^{\mathrm{reg}}(F)$. 
If $V(F) \neq V^{\mathrm{reg}}(F)$, then, by remark $6.0 .3, V^{\mathrm{reg}}(F)$ is the projection of a finite union of regular varieties $V_{i}$ still of dimension $k$, hence it follows from what we have just proved that $\mathcal{B}\left(V_{i}\right)$ is finite, and hence so is $\mathcal{B}\left(\pi\left(\bigcup V_{i}\right)\right)=$ $\mathcal{B}\left(V^{\mathrm{reg}}(F)\right)$.

Finally, if $V(F)$ is any variety, not necessarily regular, then by Theorem 5.0.7 it follows that $V(F)$ is a finite union of clopen subsets of regular sets, hence, by what we have just proved, $\mathcal{B}(V(F))$ is finite.

6.0.7 Remark (Definably connected components). Since the boolean algebra $\mathcal{B}(V(F))$ is finite, then there is an atom. If $A$ is an atom, then it is clearly a maximal definably connected subset, i.e. a definably connected component. Hence we have proved that $V(F)$ has a finite number of definably connected components.

\section{A non-analytic example}

We give an example of noetherian differential ring over the real numbers, which contains non-analytic functions. We need to give some definitions.

Let $X$ is a closed and bounded interval $[a, b] \subset \mathbb{R}$. Let $C^{\infty}(X, \mathbb{R})$ be the ring of all real functions which are $C^{\infty}$ on a neighbourhood of $X$.

7.0.1 Definition. A function $f \in C^{\infty}(X, \mathbb{R})$ is flat at the point $x_{0} \in X$ if all derivatives of $f$ vanish in $x_{0}$, but there is no neighbourhood of $x_{0}$ where $f$ assumes only the value zero. A function $f \in C^{\infty}(X, \mathbb{R})$ is flat if it is flat at some point $x_{0} \in X$.

7.0.2 Definition. A ring $M \subseteq C^{\infty}(X, \mathbb{R})$ is quasi-analytic if for every $x_{0} \in X$ the ring homomorphism

$$
\begin{aligned}
\eta: M & \rightarrow \mathbb{R}[[x]] \\
f & \mapsto \text { the Taylor expansion of } f \text { at } x_{0}
\end{aligned}
$$

is injective. Equivalently, $M$ does not contain flat functions.

7.0.3 Remark. The arguments appearing in the proof of Lemma 4.0.4 show that if $M \subseteq C^{\infty}(X, \mathbb{R})$ is noetherian and closed under differentiation, then $M$ is quasi-analytic.

7.0.4 Definition. A ring $N \subseteq C^{\infty}(X, \mathbb{R})$ is closed under taking $C^{\infty}$-quotients if the following holds: for all $f, g \in N$, if $h:=\frac{f}{g}$ is $C^{\infty}$ on $X$, then $h \in N$. 
Given a ring $M \subseteq C^{\infty}(X, \mathbb{R})$, we denote by $\bar{M}$ the closure of $M$ under $C^{\infty}$-quotients, i.e. the smallest ring containing $M$ and closed under taking $C^{\infty}$-quotients.

The following result is well known folklore.

7.0.5 Theorem. Let $M \subseteq C^{\infty}(X, \mathbb{R})$ be a quasi-analytic ring, which is closed under differentiation and contains the coordinate functions. Then its closure under $C^{\infty}$-quotients $\bar{M}$ is closed under differentiation, quasi-analytic and noetherian.

7.0.6 Remark. The theorem holds true, with the same proof, if $X=\mathbb{R}$ and all functions in $M$ have finitely many zeroes with finite multiplicities. In particular, if $M$ consists of functions which are definable in some o-minimal expansion of the real field, then $\bar{M}$ is noetherian.

7.0.7 Definition. Let $M \subset C^{\infty}([0,1], \mathbb{R})$ be a quasi-analytic ring, closed under differentiation and containing the coordinate function $x$. Let $N:=$ $\{f \uparrow(0,1) \mid f \in \bar{M}\}$ and $\mathcal{M}:=N[x]$.

$\mathcal{M} \subset C^{\infty}(\mathbb{R}, \mathbb{R})$ is clearly noetherian and closed under differentiation. Moreover, if $M$ contains a non-analytic function, then so does $\mathcal{M}$.

7.0.8 Example. A concrete example of the above construction can be obtained by taking as $M$ a quasi-analytic Denjoy-Carleman class on $[0,1]$ (according to the definition in [11]).

\section{References}

[1] L. van den Dries, Tame topology and o-minimal structures, London Mathematical Society Lecture Note Series, 248, Cambridge University Press, Cambridge, 1998.

[2] L. van den Dries, C. Miller, On the real exponential field with restricted analytic functions, Israel J. Math. 85 (1994), no. 1-3, 19-56.

[3] S. Fratarcangeli, Rolle leaves and o-minimal structures, Doctoral Thesis 2006.

[4] A. Gabrielov, Complements of subanalytic sets and existential formulas for analytic functions, Invent. Math. 125 (1996), no. 1, 1-12.

[5] F. John, Partial differential equations, Applied Mathematical Sciences, Vol. 1. Springer-Verlag, New York-Berlin, 1971. viii+221 pp. 
[6] A. G. Khovanskii, A class of systems of transcendental equations, Dokl. Akad. Nauk SSSR 255 (1980) 762-765.

[7] A. G. Khovanskii, Fewnomials, Translations of Mathematical Monographs, 88. American Mathematical Society, Providence, RI, 1991. viii+139 pp.

[8] A. Macintyre, A. Wilkie, On the decidability of the real exponential field, in: Kreiseliana, A. K. Peters, Wellesley, MA, 1996, pp. 441-467.

[9] C. Miller, Expansions of dense linear orders with the intermediate value property, J. Symbolic Logic 66 (2001), no. 4, 1783-1790.

[10] A. Pillay, C. Steinhorn, Definable sets in ordered structures. I, Trans. Amer. Math. Soc. 295 (1986), no. 2, 565-592.

[11] J.-P. Rolin, P. Speissegger, A.J. Wilkie, Quasianalytic Denjoy-Carleman classes and o-minimality, J. Amer. Math. Soc. 16 (2003), no. 4, 751-777.

[12] W. Rudin, Principles of mathematical analysis. Third edition, International Series in Pure and Applied Mathematics. McGraw-Hill Book Co., New York-Auckland-Dsseldorf, 1976. x+342 pp.

[13] G. E. Sacks, Saturated model theory, Mathematics Lecture Note Series. W. A. Benjamin, Inc., Reading, Mass., 1972. xii+335 pp.

[14] T. Servi, On the First Order Theory of Real Exponentiation, Doctoral thesis 2007.

[15] A. J. Wilkie, Model completeness results for expansions of the ordered field of real numbers by restricted Pfaffian functions and the exponential function, J. Amer. Math. Soc. 9 (1996) 1051-1094. 